\title{
Cost-effectiveness analysis of hemodialysis plus hemoperfusion versus hemodialysis alone in adult patients with end-stage renal disease in China
}

\author{
Haiyin Wang ${ }^{1 \#} \wedge$, Huajie Jin ${ }^{2 \#}$, Wendi Cheng ${ }^{1 \#}$, Xiaoxiao Qin ${ }^{1 \wedge}$, Yashuang Luo ${ }^{1 \wedge}$, Xin Liu ${ }^{1} \wedge$, Yuyan Fu ${ }^{1 \wedge}$, \\ Gengru Jiang $^{3}$, Wei Lu ${ }^{3}$, Chunlin Jin ${ }^{1}$, Mark Pennington ${ }^{2} \wedge$
}

${ }^{1}$ Health Technology Assessment Research Department, Shanghai Health Development Research Centre, Shanghai, China; ${ }^{2}$ King's Health Economics, Institute of Psychiatry, Psychology \& Neuroscience at King's College London, London, UK; ${ }^{3}$ Renal Division, Department of Internal Medicine, Xin Hua Hospital Affiliated to Shanghai Jiao Tong University School of Medicine, Shanghai, China

Contributions: (I) Conception and design: H Wang, H Jin, W Cheng, G Jiang, W Lu, C Jin, M Pennington; (II) Administrative support: X Qin, Y Luo, X Liu, Y Fu; (III) Provision of study materials or patients: G Jiang, W Lu; (IV) Collection and assembly of data: H Wang, H Jin, W Cheng, X Qin, Y Luo, X Liu, Y Fu, M Pennington; (V) Data analysis and interpretation: H Wang, H Jin, M Pennington; (VI) Manuscript writing: All authors; (VII) Final approval of manuscript: All authors.

\#These authors contributed equally to this work.

Correspondence to: Dr. Huajie Jin, PhD. King's Health Economics, Institute of Psychiatry, Psychology \& Neuroscience at King's College London, Box 024, The David Goldberg Centre, London SE5 8AF, UK. Email: huajie.jin@kcl.ac.uk; Dr. Gengru Jiang, MD, PhD. Renal Division, Department of Internal Medicine, Xin Hua Hospital Affiliated to Shanghai Jiao Tong University School of Medicine, 1665 Kongjiang Road, Yangpu District, Shanghai 200092, China. Email: jianggengru@xinhuamed.com.cn; Dr. Wei Lu, MD. Renal Division, Department of Internal Medicine, Xin Hua Hospital Affiliated to Shanghai Jiao Tong University School of Medicine, 1665 Kongiiang Road, Yangpu District, Shanghai 200092, China. Email: luweixh@126.com; Dr. Chunlin Jin, PhD. Health Technology Assessment Research Department, Shanghai Health Development Research Centre, No. 181 Xinbei Road, Shanghai 201199, China. Email: jinchunlin@shdrc.org.

Background: This study evaluates the cost-effectiveness of hemodialysis (HD) plus hemoperfusion (HP) with HD alone in adult patients with end-stage renal disease (ESRD) in China.

Methods: A Markov model was constructed to assess the cost-effectiveness of interventions over a lifetime horizon. Model parameters were informed by the HD/HP trial, the first randomized, open-label multicenter trial comparing survival outcomes and incidence of cardiovascular disease (CVD) for HD + HP versus HD alone, and supplemented by published literature and expert opinion. The primary outcome was the incremental cost-effectiveness ratio (ICER) with respect to quality adjusted life-years (QALY). The robustness of the results was examined in extensive sensitivity analyses. Analyses were conducted from a healthcare perspective. Costs were reported in both Chinese Renminbi (RMB) and US Dollars (USD) in 2019 values.

Results: The base case ICER of HD + HP is RMB 174,486 (USD 25,251) per QALY, which is lower than the RMB 212,676 (USD 30,778) willingness-to-pay threshold of three times Gross Domestic Product. This conclusion is sensitive to the mortality for patients with no severe CVD events, the incidence of CVD events, and the cost of HP and HD. At a willingness-to-pay threshold of RMB 212,676 (USD 30,778) per QALY gained, the probability that HD + HP is cost-effective is $58 \%$.

\footnotetext{
^ ORCID: Haiyin Wang, 0000-0001-9560-0460; Huajie Jin, 0000-0002-3872-3998; Wendi Cheng, 0000-0002-4198-7767; Xiaoxiao Qin, 00000001-9363-9265; Yashuang Luo, 0000-0002-4229-3249; Xin Liu, 0000-0001-9092-794X; Yuyan Fu, 0000-0002-4520-0001; Mark Pennington, 00000002-1392-8700.
} 
Conclusions: Our results indicate a potential for HD + HP to be cost-effective for patients with ESRD. Further evidence on the longer-term impact of HD + HP on CVD event rates and mortality unrelated to CVD is needed to robustly demonstrate the cost-effectiveness of HD + HP.

Trial Registration: The HD/HP trial was registered with the Chinese Clinical Trial Registry (ChiCTRIOR-16009332).

Keywords: End-stage renal disease (ESRD); cost-utility analysis; Markov model; hemodialysis (HD); hemoperfusion (HP)

Submitted Mar 09, 2021. Accepted for publication May 23, 2021.

doi: $10.21037 /$ atm-21-1100

View this article at: https://dx.doi.org/10.21037/atm-21-1100

\section{Introduction}

End-stage renal disease (ESRD) is a serious illness associated with significant health consequences and substantial financial burden. The number of ESRD patients in China was estimated at 20 million in 2017 (1). The options of renal replacement treatment for ESRD include hemodialysis (HD), peritoneal dialysis, and kidney transplant. According to the China National Hemodialysis and Peritoneal Dialysis Registry (1), the majority of patients who required renal replacement treatment received HD (86.7\%), with the remaining patients receiving peritoneal dialysis (13.3\%). Kidney transplant is rare in China due to high treatment costs and shortage of kidneys.

Although HD can efficiently remove small water-soluble uremic toxins, such as urea or parathyroid hormone, it is less efficient in removing medium or large, protein-bound uremic toxins such as phenolic or indolic compounds. The latter uremic toxins are closely associated with a high incidence of cardiovascular disease (CVD), which accounts for over $50 \%$ of all-cause mortality for patients with ESRD in maintenance HD. Hemoperfusion (HP) allows for the removal of uremic toxins by direct contact with activated charcoal or resin via adsorption. Several small-scale clinical trials have demonstrated that HD combined with HP can effectively remove small watersoluble solutes, medium-sized molecules and protein-bound uremic toxins (2-6). However, none of the previous trials directly assessed the impact of HP on clinical outcomes such as CVD events or survival. In addition, use of HP is associated with increased cost. The monthly cost of HP per patient in China is estimated to be $\$ 333$ US dollars (7). To our knowledge, none of the existing studies have assessed the cost-effectiveness of HP for patients with ESRD. In 2014, the first clinical trial which aimed to compare the survival outcomes of HD + HP with HD alone for patients with ESRD was conducted in China ("HD/HP trial", registration number: ChiCTR-IOR-16009332) (8). This study exploits data collected by the HD/HP trial along with data from the literature to evaluate the cost-effectiveness of $\mathrm{HD}+\mathrm{HP}$ as an alternative to HD alone for patients with ESRD. We present the following article in accordance with the Consensus on Health Economics Evaluation Report Standards (CHEERS) reporting checklist (available at https://dx.doi.org/10.21037/atm-21-1100) (9).

\section{Methods}

This analysis compared the cost-effectiveness of HD + HP versus HD alone for a hypothetical cohort of 54-year-old adults with ESRD in China requiring renal replacement treatment. Following the revised Brennan's toolkit (10), a Markov model was chosen to simulate the incidence, costs and outcomes of CVD events and calculate the lifetime costs and quality adjusted life expectancy. The model was parameterized using data from the HD/HP trial and published literature.

\section{HD/HP trial}

The HD/HP trial is a randomized, open-label multicenter trial which compared the clinical effectiveness and safety of $\mathrm{HD} / \mathrm{HP}$ versus HD alone for patients with ESRD in China. The protocol of the study was reported in Lu et al. (8) and is briefly summarized below. The inclusion criteria of the HD/HP trial were:

* Aged 18-75 years;

* Receiving regular blood purification treatment for at least 3 months before enrolment in the study; 


\section{* Standard $\mathrm{Kt} / \mathrm{V} \geq 1.2{ }^{1}$}

Patients were excluded if they had a life expectancy less than 1 year; had abnormal white cell count; or had major cardiovascular events in the past eight weeks. Patients were randomly allocated to the two arms with a 1:1 ratio, without stratification by patient characteristics. The following data were collected at baseline and six follow-ups $(4,12,24,48$, 72 and 96 weeks): major CVD events and cause of death (CVD or non-CVD). Quality of life was measured by the Kidney Disease Quality of Life Short Form (KDQOL$\mathrm{SF})$ at baseline and 96-week follow-up. Major CVD events included angina pectoris, myocardial infarction, severe arrhythmia, congestive heart failure, cerebral infarction, heart surgery, and peripheral vascular disease. Treatments in the HD group were specified as low-flux HD treatment at a frequency of two times a week and online hemodiafiltration treatment at a frequency of once a week, with each treatment session lasing 4 hours. Treatments in the HD + HP group were specified as all treatments in the HD group, as well as HP (HA130 HP cartridge, Jafron Biomedical Co., Ltd, China) once every 2 weeks.

The trial was conducted in accordance with the Declaration of Helsinki (as revised in 2013). The study was approved by Ethical Committee of the 30 participating centers (Xinhua Hospital, Shanghai, China; Renji Hospital Shanghai Jiao Tong University School of Medicine, Shanghai, China; Shanghai Ninth People's Hospital, Shanghai Jiao Tong University School of Medicine, Shanghai, China; Shanghai General Hospital (Songjiang District), Shanghai, China; Jinshan Hospital Affiliated to Fudan University, Shanghai, China; the Fifth People's Hospital of Shanghai, Fudan University, Shanghai, China; Changhai Hospital Affiliated to The Second Military Medical University, Shanghai, China; Tongji Hospital Affiliated Tongji University of Shanghai, Shanghai, China; Hongshan Hospital of Shanghai, Shanghai, China; Dongfang Hospital Affiliated Tongji University of Shanghai, Shanghai, China; Yangpu Hospital Affiliated to Shanghai Tong Ji University, Shanghai, China; Longhua Hospital Shanghai University of Traditional Chinese Medicine, Shanghai, China; 455 Hospital of Chinese Liberation Army, Shanghai, China; 85 Hospital of People's Liberatlon Army, Shanghai, China; Armed Police Corps Hospital of Shanghai, Shanghai, China; Shanghai
Construction Group (SCG) Hospital, Shanghai, China; Xinhua Hospital Affiliated to Shanghai Jiao Tong University School of Medicine Chongming Branch, Shanghai, China; Central Hospital of Minhang District, Shanghai, China; the Fifth People's Hospital Affiliated to Shanghai Jiao Tong University School of Medicine Songjiang Branch, Shanghai, China; Seventh People's Hospital of Shanghai University of Traditional Chinese Medicine, Shanghai, China; Changning District Central Hospital of Shanghai, Shanghai, China; Jing'an District Central Hospital of Shanghai, Shanghai, China; Tongren Hospital, Shanghai Jiaotong University School of Medicine, Shanghai, China; Renji Hospital Shanghai Jiaotong University School of Medicine, Jiading Branch, Shanghai, China; Zhabei District Central Hospital of Shanghai, Shanghai, China; the Sixth Hospital Affiliated to Shanghai Jiao Tong University School of Medicine Jinshan Branch, Shanghai, China; Shanghai Fengxian District Central Hospital, Shanghai, China; Shanghai Punan Hospital of Pudong New District, Shanghai, China; Shanghai Pudong New District Zhoupu Hospital, Shanghai, China; and the Tenth People's Hospital Affiliated to Tongji University, Shanghai, China). It has been assigned the following protocol ID: XHEC-C-2014-046-2. Written informed consent was obtained from all the study participants.

\section{Perspective and outcomes}

A costing perspective of the Chinese healthcare system was adopted. All costs were expressed in Chinese Renminbi (RMB) (2019 value) and converted to United States Dollars (USD) using the Organization of Economic Cooperation and Development (OECD) annual exchange rate for 2019 (1 USD =6.91 RMB) (11). The primary outcome was quality adjusted life-years (QALYs), which are a composite measure of quality of life and survival (12).

\section{Model structure}

Lifetime costs and outcomes were estimated using a Markov model (Figure 1) to simulate outcomes following each of the treatment strategies. The model was developed using Excel 2010 (Microsoft Corporation, US). In Figure 1, each circle represents a health state and arrows represent possible

\footnotetext{
${ }^{1} \mathrm{Kt} / \mathrm{V}$ is defined as clearance of urea multiplied by dialysis duration and normalized for urea distribution volume.
} 


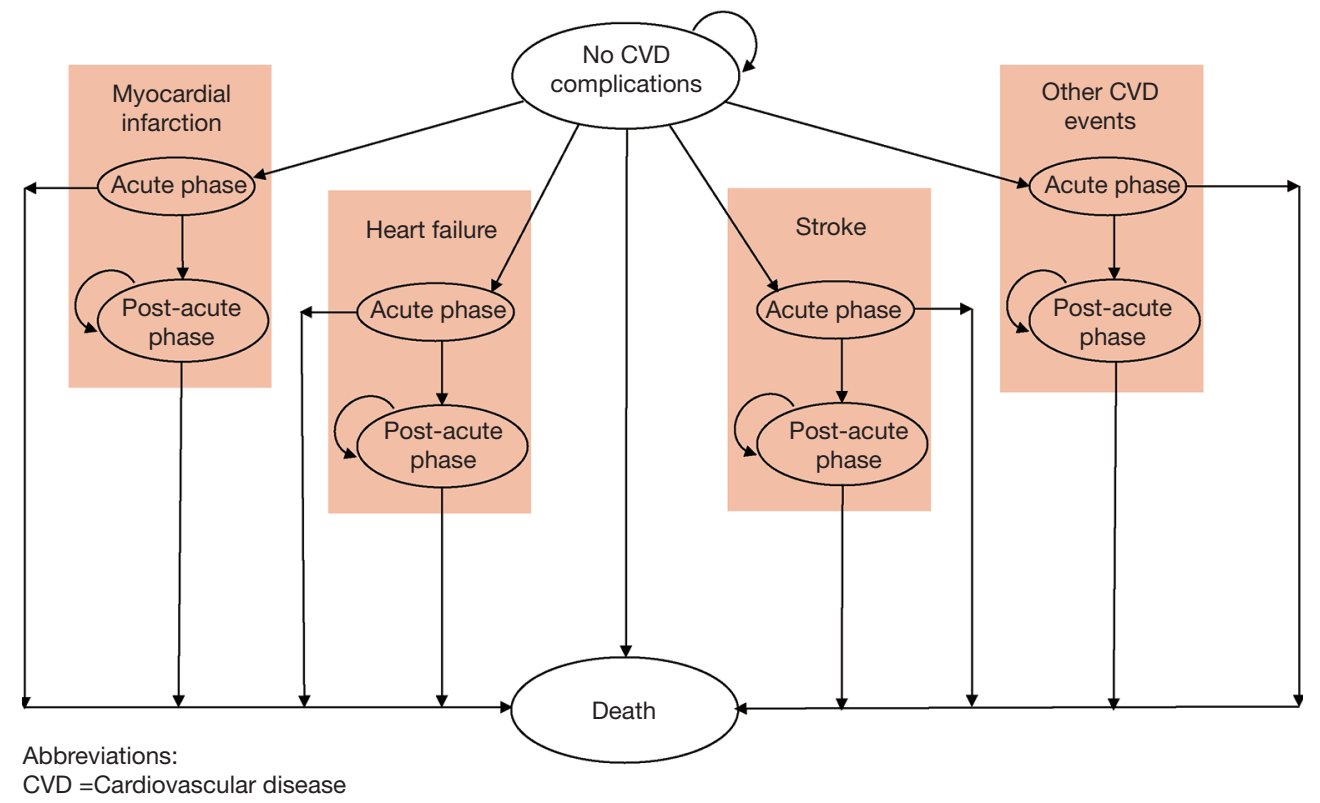

Figure 1 Model structure.

transitions at the end of each 1-month time cycle. Following treatment with either HD/HP or HD, all patients start in the "No CVD complications" health state in the model. During treatment, they may or may not experience severe CVD complications, including myocardial infarction, heart failure, stroke, and other CVD events. Patients surviving severe CVD complications progress through two stages: acute ( $0-30$ days), and post-acute (after 30 days). Each stage is associated with different mortality rates and treatment costs. All patients are at risk of death, which includes both CVD-death and non-CVD-death. The four key assumptions of the model are:

(I) The incidence of non-CVD severe complications was assumed to be the same across different treatment groups. Therefore, non-CVD severe complications were not simulated in the model.

(II) Patients continue renal replacement treatment during treatment for severe CVD complications.

(III) Deaths within three days of a CVD event were assumed to be caused by the CVD event.

(IV) The disutility of severe CVD events after the acute phase was assumed to last for a lifetime.

The three key simplifications of the model are:

(I) Patients can only experience one severe CVD complication.

(II) Patients who die from a CVD complication do not accrue any QALYs after the CVD event.
(III) The acute treatment cost of CVD complications was assumed to be the same for all patients experiencing a CVD event regardless of the survival outcome.

\section{Input data}

The model required input parameters for transitions between health states, treatments costs, and health related quality of life in each health state. The short-term (96-week) clinical effectiveness of HD/HP and HP alone were obtained from the HD/HP trial, including incidence of severe CVD events, CVD mortalities, and non-CVD mortality. The effect of treatment on quality of life and the relative risk (RR) of CVD events was estimated using regression analysis to control for patient baseline characteristics including age, sex, baseline utility value, and frequency of dialysis before entering the trial. The long-term (i.e., from week 96 onwards) rates of severe CVD events were extrapolated from short-term trial data using standard parametric distributions, following the methods suggested by Latimer (13). The model fit parameters of alternative parametric models are reported in Supplementary file (available online: https://cdn.amegroups.cn/static/public/ atm-21-1100-1.pdf), Section 1. The long-term RR of death for ESRD patients with and without severe CVD events was calculated from data on 175,840 patients 
with CKD recorded in the US Renal Data System (14). The long-term non-CVD mortality was calibrated to the reported 10 -year survival rate $(27 \%)$ for patients on maintenance HD in China (15), using the RR of death (CVD versus no CVD) obtained from the US Renal Data System.

Health state utility values for patients according to treatment arm and prior to experiencing severe CVD events were obtained from the HD/HP trial. Yang et al. (16) have published mapping algorithms from KDQOL-SF to EQ-5D tariffs for six countries: France, Germany, Italy, Spain, UK and Singapore. Mapped values for patients not experiencing severe CVD events ranged from 0.66 (French tariffs) to 0.91 (UK tariffs). Tariff values for Singapore were considered the best match for mainland China and were used in this study. After controlling for patient baseline characteristics including age, sex, baseline utility value, and frequency of dialysis before entering the trial, the mapped utility values at 96 weeks were 0.839 for the HD group and 0.844 for the HD + HP group. The Cost-Effectiveness Analysis Registry (17) was searched for literature to provide disutilities for severe CVD events (18-20). The retrieved disutilities were combined with utility by treatment arm using an additive model.

Patients accrued costs for interventions (HD alone or HD + HP), outpatient follow-ups, and treatment of severe CVD complications. Unit costs were predominantly obtained from the Shanghai healthcare reference costs 2018 (7), as no more recent unit costs were available. The frequencies of HD, HP and hemodiafiltration for each treatment group were informed by the HD/HP trial. The costs of treating acute and post-acute CVD events were estimated based on data from the China Statistical yearbook 2019 (21), the Chinese CVD clinical guideline (22), other published literature (23-26), and expert opinion. A discount rate of $5 \%$ was applied to both costs and QALYs, as recommended by the China Guideline for Pharmacoeconomic Evaluations (27). All input data for cost-effectiveness analysis are reported in Table 1.

\section{Cost-effectiveness analysis}

Patients accrued QALYs in each health state as the product of the quality of life tariff attached to the health state and the time spent in that health state. Costs and QALYs were summed over the lifetime model time horizon, after discounting. Cost-effectiveness is reported as the incremental cost-effectiveness ratio (ICER), which is the ratio of the additional cost divided by the additional effectiveness of a treatment strategy compared to the next most effective strategy. Where one strategy is more effective and less costly than a comparator, the comparator is dominated. In line with the WHO recommendations (29), (I) interventions with an ICER less than the average Chinese GDP per capita (RMB 70,892 (USD 10,259) per QALY) are considered very cost-effective, (II) interventions with an ICER less than three times GDP per capita (RMB 212,676 (USD 30,778) per QALY) are considered costeffective, and (III) interventions with an ICER exceed there times GDP per capita are considered not cost-effective.

\section{Sensitivity analysis}

Extensive sensitivity analyses were undertaken to test the robustness of the results to different sets of assumptions and different input data, including one-way sensitivity analysis, probabilistic sensitivity analysis (PSA) and structural sensitivity analysis. Sensitivity analysis assessed the impact of variation in each parameter singly across plausible ranges, and scenario analysis examined impact of variation in key parameters. The impact of joint uncertainty across all sampled parameters was examined simultaneously in PSA. A distribution reflecting underlying uncertainty was specified for each parameter, and a value sampled from the respective distribution prior to evaluating the model. Outputs from 5,000 simulations of the model allow estimation of the mean value and distribution of incremental costs and outcomes derived from Monte Carlo simulation of the joint impact of parameter uncertainty. Details on the specification of distributions for each parameter are provided in Table 1. Structural sensitivity analysis was undertaken to assess the impact of alternative modelling assumptions. In the base case, the long-term incidence rates of severe CVD events for both treatment groups were extrapolated from trial data using standard parametric distributions. In structural sensitivity analysis, the long-term incidence rates of CVD events in the HD group were obtained from published literature. The incidence of severe CVD events in Chinese patients on dialysis have been assessed by two large-scale multi-center cohort studies $(30,31)$. Of these two studies, the study conducted by Hou et al. (31) in 2012 has a larger sample size $(2,388)$ and was more recent. Therefore, the data reported by Hou et al. 2012 was tested in the structural sensitivity analysis. The long-term incidence rates of CVD events in the HD + HP group were calculated by multiplying the incidence rates in the $\mathrm{HD}$ group by the RR data taken from the HD/HP trial. 
Table 1 Summary of input data

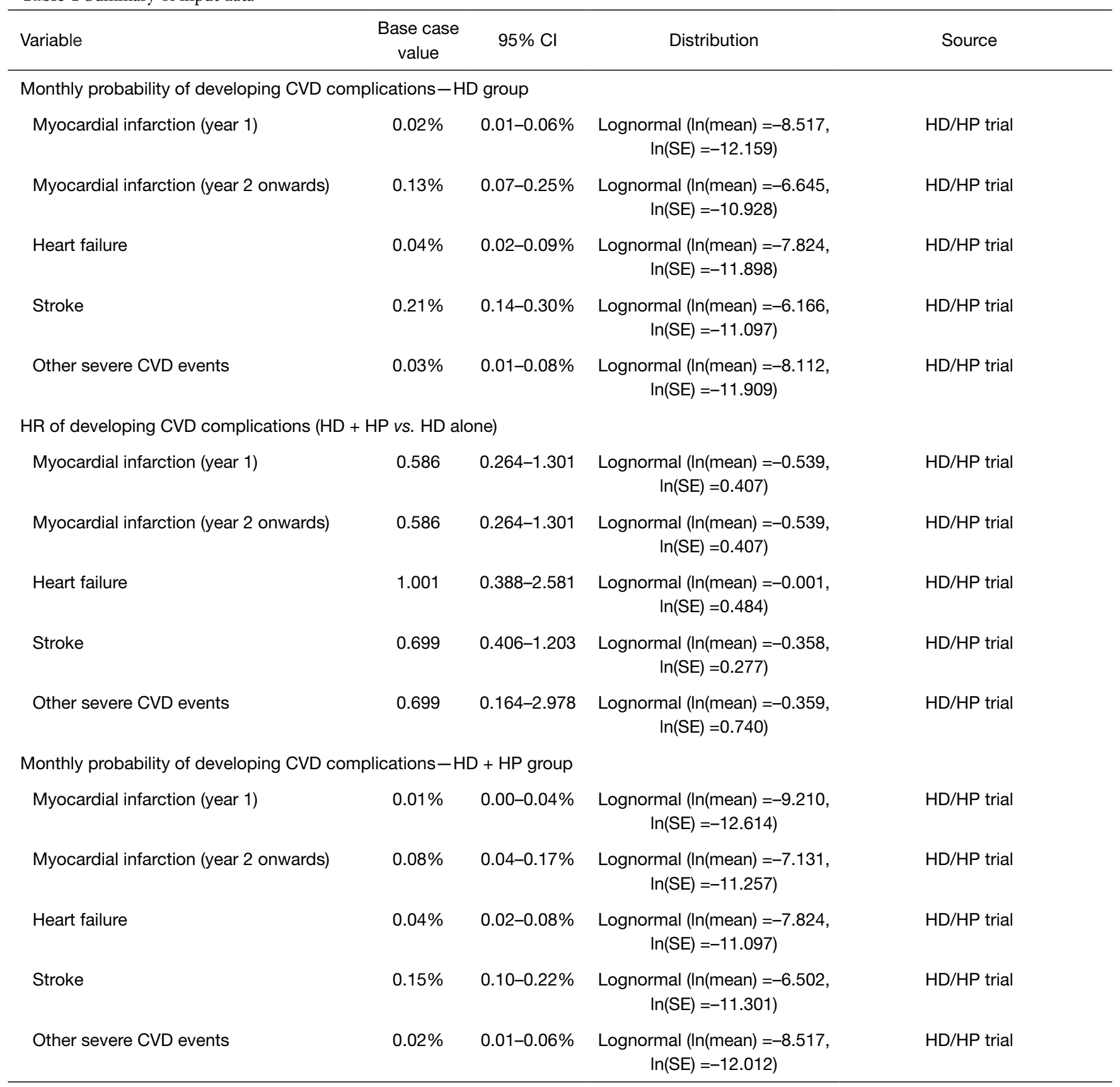

Table 1 (continued) 
Table 1 (continued)

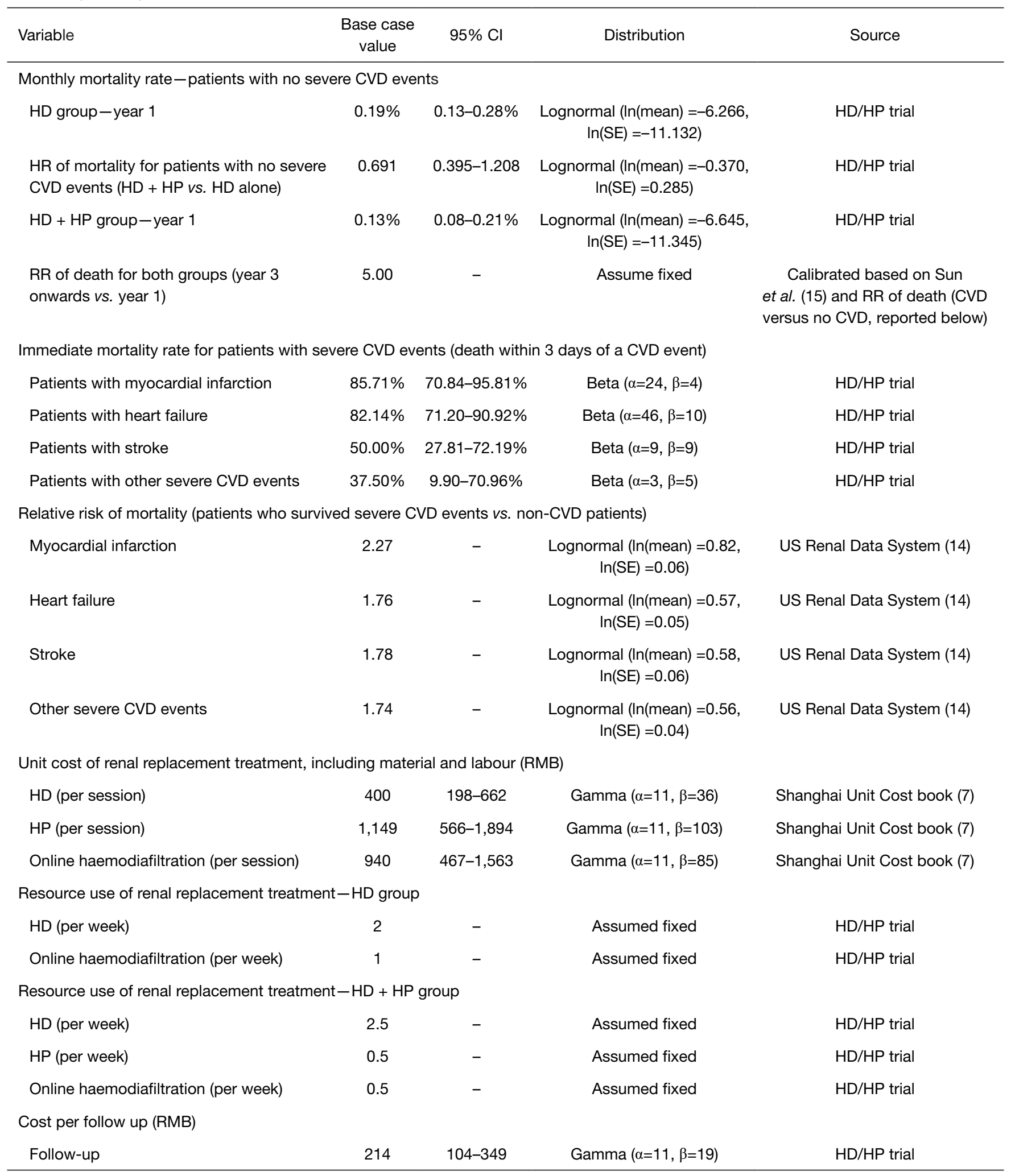

Table 1 (continued) 
Table 1 (continued)

\begin{tabular}{|c|c|c|c|c|}
\hline Variable & $\begin{array}{c}\text { Base case } \\
\text { value }\end{array}$ & $95 \% \mathrm{Cl}$ & Distribution & Source \\
\hline \multicolumn{5}{|c|}{ Cost of treating severe CVD complications - per acute episode (RMB) } \\
\hline Heart failure & 33,796 & $21,199-48,430$ & Gamma $(\alpha=23, \beta=1,454)$ & Huang et al. (23) \\
\hline \multicolumn{5}{|c|}{ Cost of treating severe CVD complications - post-acute phase, per month (RMB) } \\
\hline Myocardial infarction & 322 & $135-562$ & Gamma $(\alpha=8, \beta=39)$ & Zhao et al. (25) \\
\hline Heart failure & 1,451 & $1,144-1,735$ & Gamma $(\alpha=89, \beta=16)$ & Zhang et al. (26) \\
\hline \multicolumn{5}{|l|}{ Utility } \\
\hline $\begin{array}{l}\text { Utility for patients with no severe CVD } \\
\text { complications-HD group }\end{array}$ & 0.907 & $0.905-0.908$ & $\begin{array}{l}\text { Lognormal }(\operatorname{In}(\text { mean })=-0.098 \\
\qquad \ln (S E)=-10.206)\end{array}$ & HD/HP trial \\
\hline $\begin{array}{l}\text { The incremental impact of HP on utility of } \\
\text { patients with no severe CVD events }\end{array}$ & 0.004 & $0.001-0.006$ & $\begin{array}{l}\text { Lognormal }(\operatorname{In}(\text { mean })=-5.521 \\
\qquad \ln (\mathrm{SE})=-6.562)\end{array}$ & $\mathrm{HD} / \mathrm{HP}$ trial \\
\hline $\begin{array}{l}\text { Utility for patients with no severe CVD } \\
\text { complications-HD/HP group }\end{array}$ & 0.910 & $0.908-0.912$ & $\begin{array}{l}\text { Lognormal }(\operatorname{In}(\text { mean })=-0.094 \\
\qquad \operatorname{In}(\mathrm{SE})=-10.180)\end{array}$ & $\mathrm{HD} / \mathrm{HP}$ trial \\
\hline $\begin{array}{l}\text { Disutility of myocardial infarction-acute } \\
\text { phase }\end{array}$ & 0.147 & $0.140-0.155$ & Beta $(\alpha=1,948, \beta=11,301)$ & Kongpakwattana et al. (19) \\
\hline Disutility of heart failure-post-acute phase & 0.039 & $0.037-0.041$ & Beta $(\alpha=1,764, \beta=43,474)$ & Deng and Liu (18) \\
\hline Disutility of stroke-post-acute phase & 0.069 & $0.063-0.071$ & Beta $(\alpha=1,815, \beta=24,492)$ & Deng and Liu (18) \\
\hline $\begin{array}{l}\text { Disutility of other CVD events-post-acute } \\
\text { phase }\end{array}$ & 0.041 & $0.039-0.043$ & Beta $(\alpha=1,767, \beta=41,550)$ & Borisenko et al. (20) \\
\hline \multicolumn{5}{|l|}{ Other data } \\
\hline Discount rate for both costs and QALYs & $5.00 \%$ & - & Not varied & $\begin{array}{l}\text { China Guideline for } \\
\text { Pharmacoeconomic } \\
\text { Evaluations (27) }\end{array}$ \\
\hline
\end{tabular}

CVD, cardiovascular disease; HD, haemodialysis; HP, haemoperfusion; HR, hazard ratio; QALY, quality adjusted life-year; RR, relative risk. 
Table 2 Base case and structural sensitivity analyses for patients with ESRD

\begin{tabular}{|c|c|c|c|c|c|c|c|c|}
\hline Intervention & Cost (RMB) & LYs & QALYs & $\begin{array}{l}\text { Incremental } \\
\text { cost (RMB) }\end{array}$ & $\begin{array}{c}\text { Incremental } \\
\text { LYs }\end{array}$ & $\begin{array}{c}\text { Incremental } \\
\text { QALY }\end{array}$ & ICER (RMB) & ICER (USD) \\
\hline \multicolumn{9}{|c|}{ Base case results $^{\dagger}$} \\
\hline $\mathrm{HD}+\mathrm{HP}$ & 740,705 & 10.70 & 6.68 & 230,376 & 2.87 & 1.32 & 174,486 & 25,251 \\
\hline$H D+H P$ & 551,716 & 6.80 & 4.86 & 146,256 & 1.28 & 0.72 & 202,396 & 29,290 \\
\hline
\end{tabular}

${ }^{\dagger}$, In the base case, the long-term (i.e., 96-week onwards) incidence rates of severe CVD events for the HD and the HD + HP group were extrapolated from trial data using standard parametric distributions. For the HD group, the long-term monthly incidence rates for myocardial infarction, heart failure, stroke and other CVD events are $0.13 \%, 0.04 \%, 0.21 \%$ and $0.03 \%$, respectively. For the HD + HP group, the long-term monthly incidence rates for myocardial infarction, heart failure, stroke and other CVD events are $0.08 \%, 0.04 \%, 0.15 \%$ and $0.02 \%$, respectively. ${ }^{\ddagger}$, In structural sensitivity analysis 1 , the long-term incidence rates of severe CVD events for the HD group were obtained from Hou et al. (31). The incidence rates of severe CVD events for the HD + HP group were calculated based on the incidence rates for the HD group, and the RR of severe CVD events derived from the HD/HP trial. For the HD group, the long-term monthly incidence rates for myocardial infarction, heart failure, stroke and other CVD events are $0.39 \%, 0.93 \%, 0.14 \%$ and $0.05 \%$, respectively. For the HD + HP group, the long-term monthly incidence rates for myocardial infarction, heart failure, stroke and other CVD events are $0.25 \%, 0.74 \%$, $0.09 \%$ and $0.03 \%$, respectively. Lys, life years; ICER, incremental cost-effectiveness ratio; NMB, net monetary benefit; QALY, qualityadjusted life of years; WTP, willingness to pay threshold.

\section{Model verification and validation}

Model verification and validation steps included: checking appropriateness of the model structure and input data $(\mathrm{HJ}$, HW and MP), testing extreme values (HW), and checking the plausibility of results with clinical experts in ESRD (WL and $\mathrm{ZY}$ ).

\section{Results}

\section{Results of the HD/HP trial}

Between 2014 and 2016, 1,407 patients with ESRD were recruited to the HD/HP trial from 30 participating clinical centers in Shanghai. The baseline demographics and clinical information of the recruited patients, and the unadjusted clinical outcomes of the trial are reported in Supplementary file, Sections 2 and 3, respectively. The regression models used for estimating the incidence of severe CVD events and mortality rates are reported in Supplementary file, Section 4. Analyses of the KDQOL-SF scores indicate a significant improvement associated with HD + HP, in concordance with the analysis of mapped EQ-5D values (Supplementary file, Section 4.3, 4.4 and 5). As shown in Table 1, compared to the HD group, patients in the HD + HP group had lower monthly probabilities of myocardial infarction (HR: 0.58), stroke (HR: 0.70), other severe CVD events (HR: 0.70) and lower monthly non-CVD mortality (HR: 0.69). HD + HP did not appear to reduce probability of heart failure (HR: 1.00).

\section{Base case, structural sensitivity analysis and PSA}

Table 2 reports costs, life years (LYs), QALYs and costeffectiveness derived from the simulation model. In the base case analysis, compared with HD alone, HD + HP results in 2.87 LYs saved, 1.32 QALY gains and an additional cost of RMB 230,376 per patient. The probabilistic ICER of HD + HP is RMB 174,486 (USD 25,251) per QALY, which is lower than the RMB 212,676 (USD 30,778) willingnessto-pay threshold. Therefore, HD + HP is considered to be cost-effective. In structural sensitivity analysis (Table 2), where the long-term incidence rates of severe CVD events were obtained from a previously published cohort study (31), the ICER of HD + HP increased to RMB 202,396 (USD $29,290)$ per QALY, but was still lower than the pre-defined willingness-to-pay threshold.

The results of the PSA are illustrated in the costeffectiveness acceptability curve (CEAC) in Figure 2. The CEAC is the plot of the likelihood an intervention is costeffective as the value placed on the outcome (i.e., QALY) 


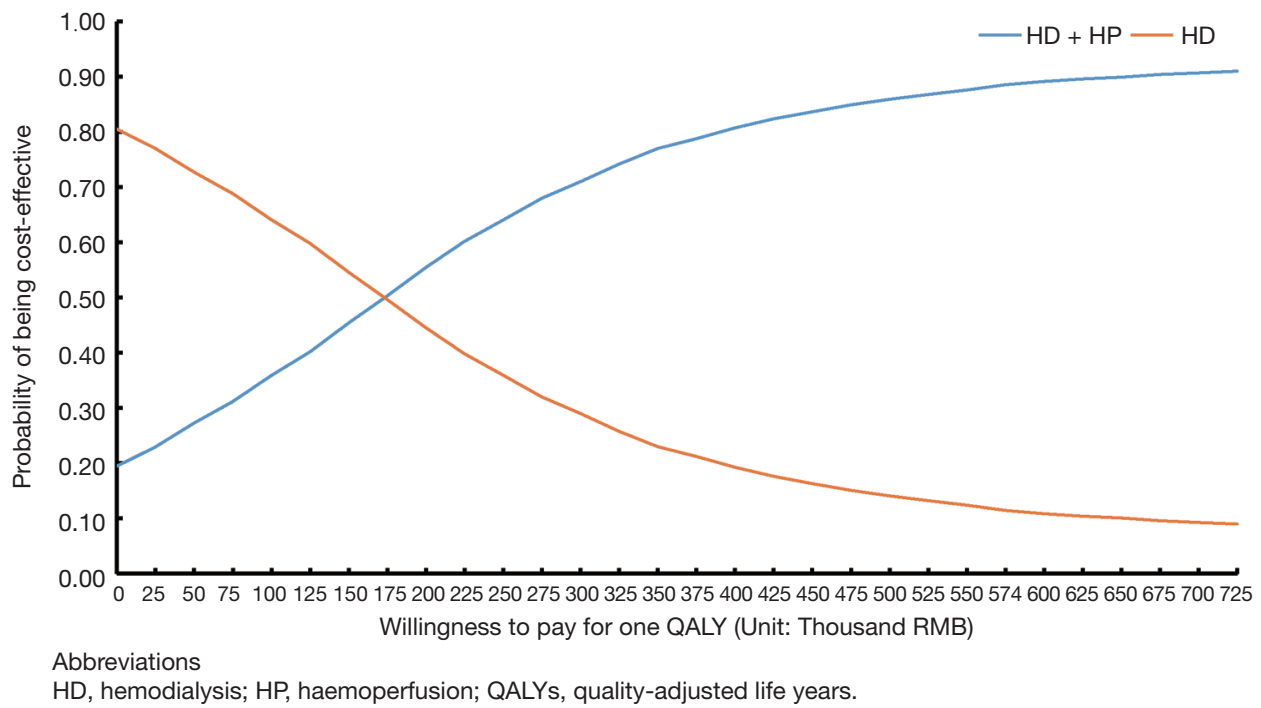

Figure 2 Cost-effectiveness acceptability curve.

is varied. At a willingness-to-pay threshold of three times GDP per capita per QALY gained (RMB 212,676/USD $30,778)$, the probability that HD + HP is cost-effective is $58 \%$. At a willingness-to-pay threshold of one times GDP per capita per QALY gained [RMB 70,892 (USD 10,259)] and two times GDP per capita per QALY gained [RMB 141,784 (USD 20,518)], the probability that HD + HP is cost-effective compared to HD alone is $30 \%$ and $44 \%$, respectively.

\section{One-way sensitivity analyses}

The results of one-way sensitivity analysis for all 39 parameters tested are reported in the Supplementary file, Section 6. The top 10 most sensitive parameters and their impacts on the results are illustrated in Figure 3. The base case conclusion (HD + HP being the most cost-effective intervention) is reversed with the following changes to parameters:

* HR of non-CVD mortality increased to 0.83 (base case value: 0.69 );

* Frequency of HP increased to 0.63 per week (base case value: 0.50 per week);

* Cost of HP increased to 1,446 RMB (base case value: $1,149 \mathrm{RMB}$ );

* Discount rate for costs reduced to $3.00 \%$ (base case value: $5.00 \%$ );

* Discount rate for QALYs increased to 6.78\% (base case value: $5 \%$ );

* Cost of HD per session increased to 566 RMB (base case value: $400 \mathrm{RMB}$ );

* Monthly incidence rate of heart failure in the HD group increased to $0.85 \%$ (base case value: $0.04 \%$ ).

\section{Discussion}

\section{The main findings and interpretation}

Our findings indicate a potential for HD + HP to be costeffective for the treatment of ESRD in China. Compared to $\mathrm{HD}$ alone, HD + HP reduces incidence of severe CVD events and subsequent CVD deaths. It is also associated with a modest improvement in quality of life and a reduction in mortality for patients with no severe CVD events. All of these effects contribute to additional QALYs for patients receiving HD + HP compared to HP alone, which are sufficient to justify the additional cost. The gain in quality of life associated with HD + HP over and above the impact on CVD events may arise from reductions in non-CVD adverse events, such as cutaneous pruritus (32) and infection (33). Further evidence on the longer-term impact of HD + HP on CVD event rates and on mortality unrelated to CVD is needed to robustly demonstrate the cost-effectiveness of HD + HP.

In structural sensitivity analysis, when the incidence rates of CVD events reported by Hou et al. (31) were used in the model, the ICER of HD + HP increased from 174,486 RMB (25,251 US dollars) to 202,396 (29,290 US dollars), but remained lower than the pre-defined willingness-to-pay 


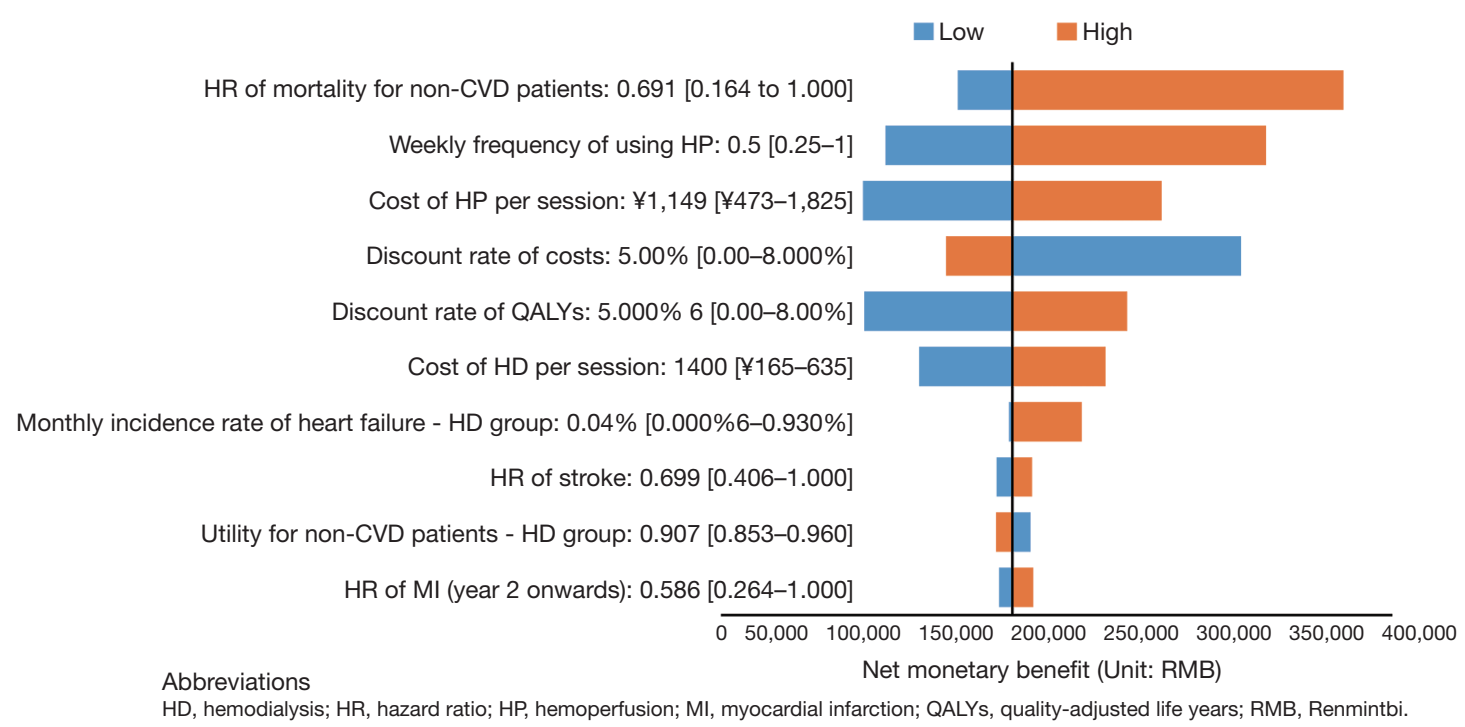

Figure 3 Result of one-way sensitivity analysis.

threshold. The reasons for an increase in the ICER are (I) the monthly incidence rate of heart failure reported by Hou et al. (31) (0.93\%) is 23 times higher than the incidence rate observed in the HD/HP trial (0.04\%); and (II) the HD/HP trial showed that use of HD + HP resulted in longer life expectancy but not reduced incidence of heart failure (HR: 1.00). Therefore, the impact of an increase in the incidence rate of heart failure was greater in the HD + HP group. Increases in the incidence rate of the other three types of CVD events (myocardial infarction, stroke and other severe CVD events) reduced the ICER for HD + HP, reflecting the reduced the incidence of myocardial infarction, stroke and other severe CVD events (HR: 0.58, 0.70 and 0.70, respectively) associated with $\mathrm{HD}+\mathrm{HP}$.

There are three reasons why the incidence rate of heart failure reported by Hou et al. (31) is much larger than the incidence rate observed in the HD/HP trial: rarity of heart failure in the trial; varying risk of CVD across China; and a healthy patient effect associated with trial enrolment. By the end of the HD/HP trial (96-week), only 18 out of 1407 patients $(1.28 \%)$ in the HD/HP trial developed heart failure. Evidence suggests the risk of CVD varies greatly across different regions in China (34). The patients in Hou et al. were recruited from six cities in China (Beijing, Shanghai, Guangzhou, Hangzhou, Wuhan, and Xian), whilst all patients in the HD/HP trial were recruited from Shanghai, which is in the region with the lowest risk of CVD (35). Hou et al. is a retrospective cohort study whilst the HD/HP trial is a randomized trial, and it has been reported that patients participating in trials experienced better outcomes compared with those outside trials $(36,37)$.

The cost-effectiveness of HD + HP was most sensitive to the HR of mortality for patients not experiencing a CVD event, rather than HD + HP's effectiveness in preventing CVD events. This reflects the low numbers of CVD events in the trial-by the end of the HD/HP trial (96-week), only $7.8 \%$ patients experienced severe CVD events, whereas non-CVD mortality was $4.2 \%$. Increased costs of HD increased the ICER for HD + HP. This is because patients in the HD + HP group received all treatments in the HD group, as well as HP, and patients in the HD + HP have a longer life expectancy than patients in the HD group. Hence patients in the HD + HP arm accrued more HD treatments.

\section{Implications for clinical practice and future research}

Our findings indicate a potential for HD + HP to be costeffective for the treatment of ESRD in China. Sensitivity analysis indicates that the cost-effectiveness of HD + HP improves for patients at higher risk of myocardial infarction, stroke and other severe CVD events. This finding supports the prioritization of HD + HP for patients at higher risk of CVD, for example, individuals who reside in northeast and north China, as those regions were reported to be associated with the highest risk of CVD (35).

The generalizability of our results to other countries is limited by two factors. Firstly, the characteristics 
of ESRD patients in China differ from those in other countries. As observed in the HD/HP trial and previous studies $(30,31,38,39)$, the leading cause of ESRD in China is chronic glomerulonephritis. In contrast, in the US and Europe, diabetes and hypertension are the main causes of ESRD $(40,41)$. In addition, the average age at commencement of dialysis in China is ten-years younger than in western countries $(30,38)$. Therefore, the risk and pattern of CVD in China might be different from other countries. Second, differences in clinical practice and treatment costs can limit the transferability of economic analysis to different countries $(42,43)$. However, our study demonstrated a potential for HD/HP to be cost-effective for patients with ESRD, and identified factors which are likely to impact on the cost-effectiveness of HD + HP. Three priorities for future research are warranted: (I) the longer-term impact of HD + HP on CVD event rates and mortality unrelated to CVD; (II) the application of a generic preference based measure, such as the EQ-5D (44), to quantify changes in patients' quality of life associated with HD + HP; and (III) exploration of the mechanisms driving improved survival and quality of life for patients receiving HD + HP.

\section{Strengths and limitations}

There are several strengths of this study. To our knowledge, this study presents the first economic analysis of the costeffectiveness of HD + HP for patients with ESRD. The analysis exploits individual patient data from the HD/ HP trial, a large clinical trial $(n=1,407)$ which is the first to assess the impact of HD + HP on incidence of severe CVD complications and mortality in patients with ESRD. Previous economic evaluations for dialysis either did not model any adverse events $(45,46)$ or only modelled general severe adverse events $(47,48)$, whilst our study explicitly modelled the cost and health impacts of four different types of severe CVD events (myocardial infarction, heart failure, stroke and other CVD events). In addition, extensive sensitivity analyses have been conducted to test the robustness of the base case inference under different assumptions and different sets of input data, in addition to capturing parameter uncertainty in a fully probabilistic model.

There are some limitations of this study, arising predominantly from limitations in the input data. First, patients recruited to the HD/HP trial were allocated to treatment using simple randomization without stratification on patient characteristics. There were some significant differences in baseline characteristics, notably sex, which might influence CVD event rates. In our analyses we adjusted for patient characteristics when estimating event rates and differences in quality of life across trial arms, which should have mitigated any risk of bias. Second, the follow-up period of the HD/HP trial is only 96 weeks, necessitating extrapolation of event rates beyond this point to capture the lifetime impact of HD + HP. A robust approach to selecting the most appropriate extrapolations was implemented (13), and the impact of alternative assumptions on the event rate beyond two years was explored. However, extrapolation of data inevitably introduces uncertainty into the analysis. Third, in the model, we assumed that patients can only experience one severe CVD complication. In reality, some patients will experience multiple, severe CVD complications during their lifetime. This simplification is likely to disfavour HD + HP, as the primary benefit of using HD + HP is to reduce the incidence of severe CVD complications. Finally, the quality of life data in the HD/HP trial were measured using KDQOL-SF rather than EQ-5D recommended by the China guideline for Pharmacoeconomic Evaluations (27). As a result, we had to use the mapping algorithm developed by Yang et al. (16) to estimate EQ-5D utility values from the KDQOL-SF scores. However, our analyses of KDQOL-SF scores indicates a significant improvement associated with $\mathrm{HD}+\mathrm{HP}$, in concordance with our analysis of mapped EQ$5 \mathrm{D}$ values.

\section{Conclusions}

Our analyses indicate a potential for HD + HP to be cost-effective for adult patients with ESRD in China. Compared to HD alone, HD + HP reduces incidence of severe CVD events and subsequent CVD deaths. It is also associated with a modest improvement in quality of life and a reduction in mortality for patients with no severe CVD events. All of these effects contribute to additional QALYs for patients receiving HD + HP compared to HP alone, which are sufficient to justify the additional cost. The costeffectiveness of HD + HP improves for patients at higher risk of myocardial infarction, stroke and other severe CVD events. Our finding supports the prioritization of HD + HP for patients at higher risk of CVD in China. Further evidence on the longer-term impact of HD + HP on CVD event rates and mortality unrelated to CVD is needed to robustly demonstrate the cost-effectiveness of HD + HP. 


\section{Acknowledgments}

The authors thank Dr. Zhenhua Yang (nephrologist consultant, Ruijin Hospital, Shanghai, China), who provided valuable advice on the clinical plausibility of the long-term clinical data extrapolated from the HD/HP trial. Funding: The work of Shanghai Health Technology Assessment Research Department (Shanghai HTA) was jointly funded by Shanghai Municipal Health Commission (Grant No. 15GWZK0901) and Jafron Biomedical Co., Ltd. The funders had no role in the study design, data collection, analysis, interpretation of data, writing, or in the decision to submit the article for publication. The views are those of the author(s) and not necessarily those of the Shanghai Municipal Health Commission or Jafron Biomedical Co., Ltd.

\section{Footnote}

Reporting Checklist: The authors have completed the CHEERS reporting checklist. Available at https://dx.doi. org/10.21037/atm-21-1100

Data Sharing Statement: Available at https://dx.doi. org/10.21037/atm-21-1100

Conflicts of Interest: All authors have completed the ICMJE uniform disclosure form (available at https://dx.doi. org/10.21037/atm-21-1100). HW, WC, XQ, YL, XL, YF, CJ report grants from Shanghai Municipal Health Commission and Jafron Biomedical Co., Ltd. MP received personal fees from Merck and from Initiate Consultancy, outside the submitted work. The other authors have no conflicts of interest to declare.

Ethical Statement: The authors are accountable for all aspects of the work in ensuring that questions related to the accuracy or integrity of any part of the work are appropriately investigated and resolved. The trial was conducted in accordance with the Declaration of Helsinki (as revised in 2013). The study was approved by Ethical Committee of the thirty participating centers (Xinhua Hospital, Renji Hospital Shanghai Jiao Tong University School of Medicine, Shanghai Ninth People's Hospital, Shanghai Jiao Tong University School of Medicine, Shanghai General Hospital (Songjiang District), Jinshan Hospital Affiliated to Fudan University, the Fifth People's Hospital of Shanghai, Fudan University, Changhai Hospital
Affiliated to The Second Military Medical University, Tongji Hospital Affiliated Tongji University of Shanghai, Hongshan Hospital of Shanghai, Dongfang Hospital Affiliated Tongji University of Shanghai, Yangpu Hospital Affiliated to Shanghai Tong Ji University, Longhua Hospital Shanghai University of Traditional Chinese Medicine, 455 Hospital of Chinese Liberation Army, 85 Hospital of People's Liberatlon Army, Armed Police Corps Hospital of Shanghai, Shanghai Construction Group (SCG) Hospital, Xinhua Hospital Affiliated to Shanghai Jiao Tong University School of Medicine Chongming Branch, Central Hospital of Minhang District, the Fifth People's Hospital Affiliated to Shanghai Jiao Tong University School of Medicine Songjiang Branch, Seventh People's Hospital of Shanghai University of Traditional Chinese Medicine, Changning District Central Hospital of Shanghai, Jing'an District Central Hospital of Shanghai, Tongren Hospital, Shanghai Jiaotong University School of Medicine, Renji Hospital Shanghai Jiaotong University School of Medicine, Jiading Branch, Zhabei District Central Hospital of Shanghai, the Sixth Hospital Affiliated to Shanghai Jiao Tong University School of Medicine Jinshan Branch, Shanghai Fengxian District Central Hospital, Shanghai Punan Hospital of Pudong New District, Shanghai Pudong New District Zhoupu Hospital, and Tenth People's Hospital Affiliated To Tongji University). Informed consent was taken from all individual participants.

Open Access Statement: This is an Open Access article distributed in accordance with the Creative Commons Attribution-NonCommercial-NoDerivs 4.0 International License (CC BY-NC-ND 4.0), which permits the noncommercial replication and distribution of the article with the strict proviso that no changes or edits are made and the original work is properly cited (including links to both the formal publication through the relevant DOI and the license). See: https://creativecommons.org/licenses/by-nc-nd/4.0/.

\section{References}

1. Zhou L, Zeng XX, Fu P. Community Hemodialysis in China: Opportunities and Challenges. Chinese Medical Journal 2017;130:2143.

2. Chen SJ, Jiang GR, Shan JP, et al. Combination of maintenance hemodialysis with hemoperfusion: a safe and effective model of artificial kidney. Int J Artif Organs 2011;34:339-47.

3. Li WH, Yin YM, Chen H, et al. Curative effect of 
neutral macroporous resin hemoperfusion on treating hemodialysis patients with refractory uremic pruritus. Medicine (Baltimore) 2017;96:e6160.

4. Raine A, Cordonnier D, Ritz E. Effect of hematodialysis plus hemoperfusion on insulin resistance and nutritional status of patients with end-stage diabetic nephropathy. J Int Transl Med 2015;51:180-4.

5. Zhang J, Yuan Y, An X, et al. Comparison of combined blood purification techniques in treatment of dialysis patients with uraemic pruritus. Int J Clin Exp Med 2016;9:8563-8.

6. Zhang Y, Mei CL, Rong S, et al. Effect of the Combination of Hemodialysis and Hemoperfusion on Clearing Advanced Glycation End Products: A Prospective, Randomized, Two-Stage Crossover Trial in Patients Under Maintenance Hemodialysis. Blood Purif 2015;40:127-32.

7. The Health Commission of Shanghai. Price of healthcare services provided by health care providers in Shanghai, 2018.

8. Lu W, Jiang GR. Randomised, open-label, multicentre trial comparing haemodialysis plus haemoperfusion versus haemodialysis alone in adult patients with end-stage renal disease (HD/HP vs HD): study protocol. BMJ Open 2018;8:e022169.

9. Husereau D, Drummond M, Petrou S, et al. Consolidated Health Economic Evaluation Reporting Standards (CHEERS)--explanation and elaboration: a report of the ISPOR Health Economic Evaluation Publication Guidelines Good Reporting Practices Task Force. Value Health 2013;16:231-50.

10. Jin H, Robinson S, Shang W, et al. Overview and Use of Tools for Selecting Modelling Techniques in Health Economic Studies. Pharmacoeconomics 2021;39:757-70.

11. OECD. Exchange rates. 2020. Available online: https:// data.oecd.org/conversion/exchange-rates.htm

12. Prieto L, Sacristán JA. Problems and solutions in calculating quality-adjusted life years (QALYs). Health Qual Life Outcomes. 2003;1:80.

13. Latimer NR. Survival analysis for economic evaluations alongside clinical trials--extrapolation with patient-level data: inconsistencies, limitations, and a practical guide. Med Decis Making 2013;33:743-54.

14. Saran R, Robinson B, Abbott K, et al. 2018 USRDS Annual Data Report: Epidemiology of kidney disease in the United States. Am J Kidney Dis 2019;73:A7-8.

15. Sun Y, Wang Y, Yu W, et al. Association of Dose and Frequency on the Survival of Patients on Maintenance of Hemodialysis in China: A Kaplan-Meier and CoxProportional Hazard Model Analysis. Med Sci Monit 2018;24:5329-37.

16. Yang F, Wong CKH, Luo N, et al. Mapping the kidney disease quality of life 36-item short form survey (KDQOL-36) to the EQ-5D-3L and the EQ-5D$5 \mathrm{~L}$ in patients undergoing dialysis. Eur J Health Econ 2019;20:1195-206.

17. Center for the Evaluation of Value and Risk in Health. The Cost-Effectiveness Analysis Registry [Internet]. (Boston), Institute for Clinical Research and Health Policy Studies, Tufts Medical Center. 2020.

18. Deng Q, Liu W. Multilevel analysis of health related quality of life of patients with cardiovascular disease and its determinants. Journal of Shandong University (Health Sciences) 2020;58:115-21.

19. Kongpakwattana K, Ademi Z, Chaiyasothi T, et al. Cost-Effectiveness Analysis of Non-Statin LipidModifying Agents for Secondary Cardiovascular Disease Prevention Among Statin-Treated Patients in Thailand. Pharmacoeconomics 2019;37:1277-86.

20. Borisenko O, Lukyanov V, Ahmed AR. Cost-utility analysis of bariatric surgery. Br J Surg 2018;105:1328-37.

21. National Bureau of Statistics of China. China Statistical yearbook 2019. Beijing, China: China Statistics Press; 2020.

22. Beijing Hypertension Association. Guidance on the management of cardiovascular disease in primary care 2020. Chinese Journal of the Frontiers of Medical Science (Electronic Version) 2020;12:1-73.

23. Huang J, Yin H, Zhang M, et al. Understanding the economic burden of heart failure in China: impact on disease management and resource utilization. J Med Econ 2017;20:549-53.

24. He N, Zhang Y, Yuan Z, et al. The prevalence and healthcare cost of patients with type 2 diabetes comorbidities in China. Chinese Journal of Endocrinology and Metabolism 2019;35:200-5.

25. Zhao X, Zhang X, Xie Q, et al. Study of Expenses on Coronary Heart Disease in Beijing Based on Volume Data. Chinese Health Economics 2017;36:63-8.

26. Zhang Y, Zhai T, Chai P, et al. Cost of cardiovascular and cerebrovascular disease in China. Chinese Health Economics 2019;5:18-22.

27. Research Group of the "China Guidelines for Pharmacoeconomic Evaluation". China Guidelines for Pharmacoeconomic Evaluations. 2019.

28. De Smedt D, Annemans L, De Backer G, et al. Costeffectiveness of optimized adherence to prevention 
guidelines in European patients with coronary heart disease: Results from the EUROASPIRE IV survey. Int J Cardiol 2018;272:20-5.

29. Bertram MY, Lauer JA, De Joncheere K, et al. Costeffectiveness thresholds: pros and cons. Bull World Health Organ 2016;94:925-30.

30. Hou FF, Ma ZG, Mei CL, et al. Cardiovascular disease in Chinese chronic renal insufficiency patients-epidemiology survey. Zhonghua Yi Xue Za Zhi 2005;85:458-63.

31. Hou F, Jiang J, Chen J, et al. China collaborative study on dialysis: a multi-centers cohort study on cardiovascular diseases in patients on maintenance dialysis. BMC Nephrol 2012;13:94.

32. Simonsen E, Komenda P, Lerner B, et al. Treatment of Uremic Pruritus: A Systematic Review. Am J Kidney Dis 2017;70:638-55.

33. Shima H, Kimura T, Nishiuchi T, et al. Successful treatment of direct hemoperfusion with polymyxin B-immobilized fiber for septic shock and severe acute kidney injury due to ceftriaxone-resistant Escherichia coli: a case report with literature review. Renal Replacement Therapy 2020;6:16.

34. Ma LY, Chen WW, Gao RL, et al. China cardiovascular diseases report 2018: an updated summary. J Geriatr Cardiol 2020;17:1-8.

35. Li X, Wu C, Lu J, et al. Cardiovascular risk factors in China: a nationwide population-based cohort study. Lancet Public Health 2020;5:e672-81.

36. Heneghan C, Goldacre B, Mahtani KR. Why clinical trial outcomes fail to translate into benefits for patients. Trials 2017;18:122.

37. Nijjar SK, D'Amico MI, Wimalaweera NA, et al. Participation in clinical trials improves outcomes in women's health: a systematic review and meta-analysis. BJOG 2017;124:863-71.

38. Hou FF, Ma ZG, Mei CL, et al. Epidemiology of cardiovascular risk in Chinese chronic kidney disease

Cite this article as: Wang $\mathrm{H}$, Jin $\mathrm{H}$, Cheng W, Qin $\mathrm{X}$, Luo Y, Liu X, Fu Y, Jiang G, Lu W, Jin C, Pennington M. Cost-effectiveness analysis of hemodialysis plus hemoperfusion versus hemodialysis alone in adult patients with end-stage renal disease in China. Ann Transl Med 2021;9(14):1133. doi: 10.21037/atm-21-1100 patients. Zhonghua Yi Xue Za Zhi 2005;85:753-9.

39. Zuo L, Wang M. Current burden and probable increasing incidence of ESRD in China. Clin Nephrol 2010;74 Suppl 1:S20-2.

40. van den Brand JAJG. Diabetes mellitus as a cause of endstage renal disease in Europe: signs of improvement. Clin Kidney J 2016;9:454-6.

41. Bikbov B, Purcell CA, Levey AS, et al. Global, regional, and national burden of chronic kidney disease, 1990\&\#x2013;2017: a systematic analysis for the Global Burden of Disease Study 2017. Lancet 2020;395:709-33.

42. Anderson R. Systematic reviews of economic evaluations: utility or futility? Health Econ 2010;19:350-64.

43. Drummond M, Sorenson C. Nasty or nice? A perspective on the use of health technology assessment in the United Kingdom. Value Health 2009;12 Suppl 2:S8-13.

44. Herdman M, Gudex C, Lloyd A, et al. Development and preliminary testing of the new five-level version of EQ-5D (EQ-5D-5L). Qual Life Res 2011;20:1727-36.

45. Wong CKH, Chen J, Fung SKS, et al. Lifetime costeffectiveness analysis of first-line dialysis modalities for patients with end-stage renal disease under peritoneal dialysis first policy. BMC Nephrol 2020;21:42.

46. Yang F, Lau T, Luo N. Cost-effectiveness of haemodialysis and peritoneal dialysis for patients with end-stage renal disease in Singapore. Nephrology (Carlton) 2016;21:669-77.

47. Teerawattananon Y, Mugford M, Tangcharoensathien $\mathrm{V}$. Economic evaluation of palliative management versus peritoneal dialysis and hemodialysis for end-stage renal disease: evidence for coverage decisions in Thailand. Value Health 2007;10:61-72.

48. Surendra NK, Abdul Manaf MR, Hooi LS, et al. Cost utility analysis of end stage renal disease treatment in Ministry of Health dialysis centres, Malaysia: Hemodialysis versus continuous ambulatory peritoneal dialysis. PLoS One 2019;14:e0218422. 